\title{
ANALYSIS OF MIXING IN A TWIN-CAM MIXER USING SMOOTHED PARTICLE HYDRODYNAMICS
}

\author{
Martin Robinson ${ }^{1}$, Paul Cleary ${ }^{2}$ and Joseph Monaghan ${ }^{1}$ \\ ${ }^{1}$ School of Mathematical Sciences, Monash University, Clayton, Victoria 3169, AUSTRALIA \\ ${ }^{2}$ CSIRO Mathematical and Information Sciences, Clayton, Victoria 3169, AUSTRALIA
}

\begin{abstract}
Numerical simulations of fluid flow in typical industrial mixers are made difficult due to geometries involving moving parts, small gaps and sharp corners. Gridded methods such as finite elements need to constantly regenerate the mesh to accommodate the moving objects, and these meshes often need to be further refined to correctly model fluid flow near small gaps and sharp corners. Smoothed Particle Hydrodynamics (SPH), a grid free particle method, does not suffer from these drawbacks associated with grids. Additionally, the Lagrangian nature of SPH is better suited to the analysis of mixing. Each particle represents the same finite volume of fluid over time, so data describing the transport of any "blob" of fluid is readily available. This paper describes 2D SPH simulations of a twin-cam mixer and compares the results against experimental data and results from two published finite-element simulations. We also present a method for calculating the Finite-Time Lyapunov Exponent (FTLE), using SPH particle data, for each particle at each timestep. These Finite-Time Lyapunov Exponents build up a picture that describes where mixing is either promoted or inhibited in the flow. The purpose of the paper is to evaluate the effectiveness of SPH and FTLEs in simulating and analysing the mixing in a typical industrial mixer.
\end{abstract}

\section{NOMENCLATURE}

$C$ right Cauchy-Green deformation tensor

$\sigma \quad$ Lyapunov Exponent

$\sigma_{t_{0}}^{T}$ Finite-Time Lyapunov Exponent

$W$ SPH kernel

$h \quad$ SPH smoothing length

$m_{a}$ mass of SPH particle a

$c_{a}$ sound speed at particle a

$r_{a b}$ distance vector from particle $\mathrm{a}$ to $\mathrm{b}$

$\mathrm{P}$ pressure

$\rho$ density

$v$ velocity

$\mu \quad$ dynamic viscosity

$c_{S} \quad$ sound speed

$M$ mixing measure

$\|x\|$ standard vector $\mathrm{L}_{2}$-norm of $x$

\section{INTRODUCTION}

\section{Smoothed Particle Hydrodynamics}

Smoothed Particle Hydrodynamics (SPH) is a Lagrangian method for numerically solving the equations of fluid dynamics. Instead of a mesh it uses particles, which are both interpolation points for the fluid variables as well as physical "blobs" of fluid. Due to its Lagrangian nature, it is well suited to modelling problems where the transport of mass is an important issue. Two or more fluids are also simple to model, as each fluid is represented by its own set of particles, which is constant throughout the simulation. Fluids with different physical properties can be modelled by changing the properties of individual particles. It is also trivial to incorporate any sort of moving boundary with SPH. Traditional meshed methods need to either re-mesh the domain at every timestep or use an additional method such as the Lagrange multiplier based fictitious domain method (See Bertrand et al 1997). SPH boundaries are constructed from particles, which can be easily moved each timestep with no additional calculation. Please see Monaghan (2005) for a good review of SPH theory.

\section{Twin Cam Mixer Experiments and Simulations}

The experimental validation in this paper uses data from the work of Avalosse and Crochet (1997). They compared their experimental results of a twin cam mixer with a $2 \mathrm{D}$ finite-element simulation. Their base mesh was a regular grid in circular coordinates, with a constant angular resolution, and with the radial resolution higher near the circle formed by the rotating tips of the triangular cams. The elements that intersected the straight edges of the cams were re-meshed at every timestep using an automatic algorithm based on Delaunay triangulation. Bertrand et al. (2003) modelled a similar 2D Twin Cam simulation using finite elements. However, instead of using a standard finite element method that requires re-meshing at every timestep, they used the Lagrange multiplier based fictitious domain method. This was combined with an adaptive mesh refinement at each timestep, which was applied around the cam tips. Results tracing the dispersion of a dyed blob of fluid for the experiment and the two simulations mentioned above are shown in Figure 3. Note that the coloured blob of material in the experiment is not completely rectangular, unlike the blob initialised in the finite-element simulations. So these simulations start with some initial error.

\section{Measuring Mixing}

Ottino (1989) has developed a popular mixing theory, which is based on the calculation of several variables along the paths of material points in the flow. He has proposed a commonly used measure of mixing strength: length stretch $\chi$. Avalosse and Crochet (1997) apply this measure to their simulation of the twin cam mixer. Let $d X$ be a material length with orientation $M$ that deforms into $d x$ with orientation $m$ after time $T$. If $C$ is the right Cauchy-Green deformation tensor then: 


$$
\chi(X, M, t)=\frac{|d x|}{|d X|}=(M \bullet C M)^{1 / 2}
$$

Ottino's length stretch is directly related to the chaos theory notion of Lyapunov exponents $\sigma$, which is a measure of how quickly a material trajectory diverges from its initial conditions. The Lyapunov exponent is related to the length stretch by

$$
\sigma=\lim _{T \rightarrow \infty} \frac{1}{T} \ln \chi
$$

As with length stretch, high values of $\sigma$ indicate a higher quality of mixing at that point. While Lyapunov exponents are only useful for periodic flows, the concept has been extended to finite times (Finite Time Lyapunov Exponents, FTLE) and finite sizes (FSLE) (Artale 1997, Pierrehumbert, 1993, Lapeyre 2002, Joseph and Legras 2002). We have used FTLEs, rather than length stretch, to study the twin cam simulation. Due to the very large values of length stretch, a logarithmic scale must be used anyway, and dividing by $T$ eliminates a scaling by the $T$ used to calculate the length stretch.

\section{Chaotic Transport}

One of the important features of a chaotic system are its stable and unstable invariant manifolds and their associated hyperbolic or stagnation points. Knowledge of the location and movement of these features gives important insight into the mixing and transport of the system (see Holmes, 1990, Rom-Kedar, 1990 or Ottino, 1989).

A brief (and very qualitative) description of a stable manifold is that a pair of particles straddling the manifold will separate faster than any other nearby pairs (except of course for a pair across the same manifold). An unstable manifold is similar to the stable version, except that the particles separate in reverse time. That is, they approach the manifold faster than any other pairs in the vicinity. Stable manifolds are associated with stretching in the flow and unstable manifold cause folding (Haller 2000).

FTLE are often used to find these manifolds when only finite-time data is available, since maxima in the forwardtime FLTE map correspond with stable manifolds and maxima in backwards-time FTLE indicate unstable manifolds.

\section{SIMULATION DESCRIPTION}

\section{Equations}

All the equations in this section, along with additional explanation, can be found in Monaghan (2005). The most commonly used kernel for SPH simulations (and the kernel used in this simulation) is the cubic spline. This has the form:

$$
W(x, h)=\frac{1}{h^{2}} \frac{15}{14 \pi}\left\{\begin{array}{cc}
{\left[(2-q)^{3}-4(1-q)^{3}\right],} & \text { for } 0 \leq q \leq 1 \\
(2-q)^{3}, & \text { for } 1<q \leq 2 \\
0, & \text { for } q>2
\end{array}\right.
$$

where $q=r / h$ and $h$ is the smoothing length of the particle. The kernel determines how the mass of particle is spread out in space, as well as how the fluid variables are interpolated between particles.

Ignoring gravity, the SPH version of the momentum equation for particle $a$ is:

$$
\frac{d v_{a}}{d t}=-\sum_{b} m_{b}\left(\frac{P_{b}}{\rho_{b}^{2}}+\frac{P_{a}}{\rho_{a}^{2}}+\prod_{a b}\right) \nabla_{a} W_{a b}
$$

where $\Pi_{\mathrm{ab}}$ is a viscosity term between particles $a$ and $b$. There are a number of forms for this viscosity term. We are using the form:

$$
\begin{aligned}
& \Pi_{a b}=-\frac{\alpha v_{s i g}\left(v_{a b} \cdot r_{a b}\right)}{\bar{\rho}_{a b}\left|r_{a b}\right|} \\
& v_{\text {sig }}=c_{a}+c_{b}-\beta v_{a b} \cdot \hat{r}_{a b}
\end{aligned}
$$

where $\beta \approx 4$ and $\alpha$ is related to the dynamic viscosity $\mu$ by:

$$
\alpha=\frac{114 \mu}{15 \rho c_{s} h}
$$

The standard form of the continuity equation for constant $h$ is:

$$
\frac{d \rho_{a}}{d t}=\sum_{b} m_{b} v_{a b} \cdot \nabla_{a} W_{a b}
$$

The equation of state models a nearly incompressible fluid, and is given by:

$$
P_{a}=B\left(\left(\frac{\rho_{a}}{\rho_{0}}\right)^{\gamma}-1\right)
$$

where $\gamma=7$. The pressure scale factor $B$ is given by:

$$
B=\frac{100 v_{\max }^{2} \rho_{0}}{\gamma}
$$

where $v_{\max }$ is an estimate of the maximum flow velocity.

The tank walls and triangular cams are modelled using boundary particles, which are not allowed to move freely but exert boundary forces on the other SPH particles. The boundary force on particle $a$ from a boundary particle $k$, where $x$ is the tangential distance (i.e. along the boundary) between $k$ and $a$ and $y$ is the normal distance between $k$ and $a$, is given by

$$
f_{a k}=-\frac{m_{a}}{m_{a}+m_{k}} B(x, y) n_{k}
$$

where $n_{k}$ is the unit normal vector to the boundary. $B$ is chosen so that it increases rapidly for small y (so as to prevent the particle from penetrating the wall) and so its variation with $x$ ensures that the particle feels no change in force as it moves tangentially along the boundary. See Monaghan (2005) for the form of $B$ used here.

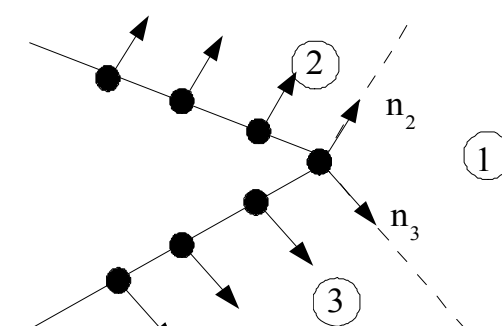

Figure 1: Multiple boundary normals for sharp corners. For a fluid particle in region 2 , use $\mathrm{n}_{2}$. In region 3 use $\mathrm{n}_{3}$. In region 1 use unit radial vector in the direction from the corner particle to the fluid particle.

Finding the correct normal vector $n_{k}$ can be difficult near sharp corners in the boundary. Large changes in the direction of neighbouring boundary normals can lead a very small value of $y$ (since $\left.y=\left|n_{k} \bullet r_{a b}\right|\right)$, which results in an unnaturally large boundary force. To fix this problem, each sharp point is represented with a particle that has two normals equal to those of its neighbouring boundary particles. The correct $n_{k}$ is calculated from these normals 
and the position of the interacting fluid particle (See Figure 1 and Monaghan 2003).

\section{Geometry}

A diagram of the twin cam geometry is shown in Figure 2. The fluid used for the experimental results was a Newtonian aqueous solution of glucose, with a viscosity of $50 \mathrm{~Pa} \mathrm{~s}$ and a density of $1500 \mathrm{~kg} / \mathrm{m}^{3}$. The counterclockwise rotating cams are moving at $0.5 \mathrm{rpm}$, which gives a Reynolds number of the order of 0.001 .

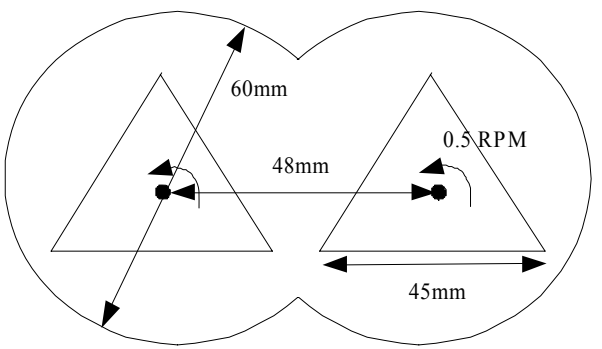

Figure 2: Geometry of Twin Cam mixer

\section{MIXING ANALYSIS}

\section{Finite-Time Lyapunov Exponent}

The FTLE is calculated considering the trajectories of particles over a finite-time period T. What follows is a brief derivation of the FTLE, please see the excellent description by Shadden (2005) for a more in depth discussion.

Consider a flow function that maps particles at time $t_{0}$ to their advected positions at time $t_{0}+T$

$$
x \mapsto \phi_{t_{0}}^{t_{0}+T}(x)
$$

Let $\delta x(t)$ be the separation of two neighbouring particles in the flow. After a time $T$, their separation can be approximated by a first-order Taylor expansion of the flow function

$$
\delta x\left(t_{0}+T\right)=\frac{d \phi_{t_{0}}^{t_{0}+T}(x)}{d x} \delta x\left(t_{0}\right)+\mathrm{O}\left(\left\|\delta x\left(t_{0}\right)\right\|^{2}\right)
$$

The magnitude of this separation is given by

$$
\begin{aligned}
& \left\|\delta x\left(t_{0}+T\right)\right\|=\sqrt{\delta x\left(t_{0}\right)^{*} C \delta x\left(t_{0}\right)} \\
& C=\frac{d \phi_{t_{0}}^{t_{0}+T}}{d x} \frac{d \phi_{t_{0}}^{t_{0}+T}}{d x}
\end{aligned}
$$

where $C$ is a finite-time version of the right Cauchy-Green deformation tensor. The maximum separation of the two particles will occur when they are aligned along the eigenvector associated with the largest eigenvalue of $C$. Thus, if $\lambda_{\max }$ is the maximum eigenvalue of $C$

$$
\max _{\delta x\left(t_{0}\right)}\left\|\delta x\left(t_{0}+T\right)\right\|=\sqrt{\lambda_{\max }}\left\|\overline{\delta x\left(t_{0}\right)}\right\|
$$

where the bar over $\delta x\left(t_{0}\right)$ indicates that it is aligned with the eigenvector associated with $\lambda_{\max }$. This can be written

$$
\max _{\delta x\left(t_{0}\right)}\left\|\delta x\left(t_{0}+T\right)\right\|=e^{\sigma_{t_{0}}^{T}(x) T}\left\|\overline{\delta x\left(t_{0}\right)}\right\|
$$

where $\sigma$ is the FTLE. That is, the maximum exponential separation speed between two neighbouring particles

$$
\sigma_{t_{0}}^{T}(x)=\frac{1}{|T|} \ln \sqrt{\lambda_{\max }}
$$

Grid-based numerical methods use tracer particles to calculate the FTLE. Since the tracers can be initialised on a regular grid, finite-differencing can be used to estimate the gradient of the flow map $\frac{d \phi_{t_{0}}^{t_{0}+T}}{d x}$. In an SPH simulation, the particles will not be regularly spaced. However, the gradient can be found by solving

$$
\delta x\left(t_{0}+T\right)=\frac{d \phi_{t_{0}}^{t_{0}+T}(x)}{d x} \delta x\left(t_{0}\right)
$$

using a least-squares method (we have used singularvalued decomposition). The data points for this equation are all the particles within the smoothing length $2 h$ of the base particle (i.e. the particle where the FTLE is being calculated). It is reasonable to assume that the gradient of the flow map is constant over this area.

\section{SIMULATION RESULTS}

\section{Experimental Validation}

The results of the simulation can be validated against the experiment performed by Avalosse and Crochet (1997). Figure 3 shows the initial setup for both the SPH simulation and experiment. The same figure also shows the simulation and experiment after the cams have rotated through $2 \pi$ and $7 \pi / 3$ radians (120 and $160 \mathrm{~s}$ ).

The motion of the cams divides the coloured blob into two segments along a line that is roughly diagonal from the lower tip of the left cam to the top of the right cam (see Figure 5, top, for an image of this line). There is a stationary point at the centre of the domain, so particles near this point have a lower velocity. This causes a trail of particles behind the bulk of the coloured particles as they leave the centre of the domain, which shortens to form a "kink" in the blob (seen most clearly in lower region of the leftmost cam at $t=120 \mathrm{~s}$ ). The blob is stretched out as it is moved around the chamber, due to lower velocities near the walls of the chamber. Once the leading edges of the divided blobs have completed a revolution and are once again between the cams, both are further divided along the same line as the initial division, and the mixing process continues. The transport of the coloured blob of fluid follows the experiment well. Comparing the results of the SPH simulation against the two finite element simulations (in the same Figure 3), the results of the SPH simulation are clearly more accurate than the simulations of Avalosse and Crochet (1997), and compare well against the results given by Bertrand et. al. (2003).

The SPH simulations shown in Figure 3 use 7995 particles (160 particles along the horizontal). Bertrand initially uses 2388 finite elements to represent the domain, but this is before the mesh refinement around the cam edges. Using a rough visual estimate, the total number of elements was approximately 6000 , which is comparable to the number of SPH particles used in these simulations.

\section{Timestep and Viscosity Issues}

Due to the very low Reynolds number of the experiment, the time-scale of the viscous forces present in the simulation is much smaller than the time-scale of the rotating cams. Estimating the viscous time scale as equal to that of a cylindrical Couette flow (with the distance between the cylinders as $d=10 \mathrm{~mm}$ ) and the time scale of the rotating cams as their period $\left(t_{\text {cam }}=120 \mathrm{~s}\right)$ gives:

$$
\frac{t_{\text {visc }}}{t_{\text {cam }}}=\frac{\rho d^{2} / \pi^{2} \mu}{120} \approx 2.5 \times 10^{-6}
$$



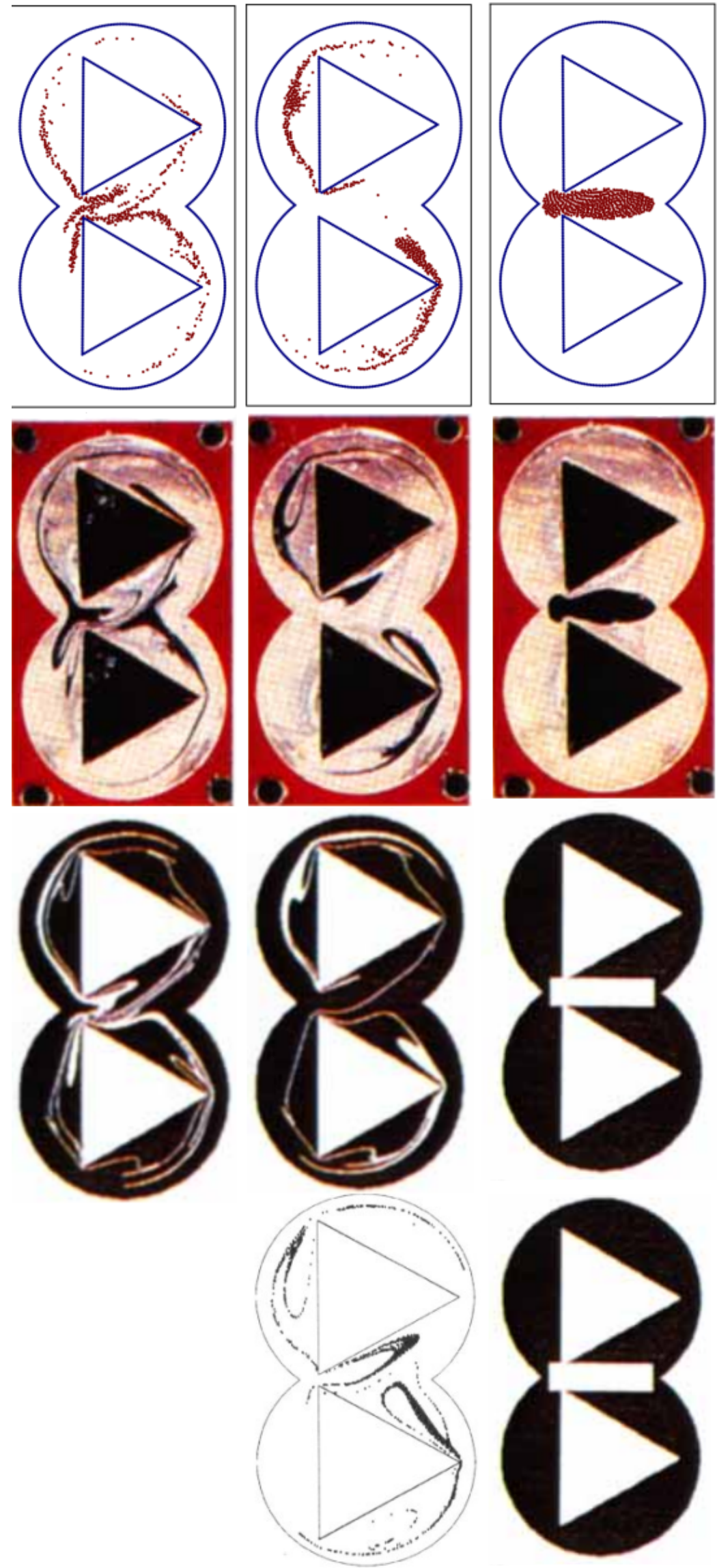

Figure 3: Comparison of SPH simulation (Top row), experiment by Avalosse \& Crochet (1997) (Second row), simulation by Avalosse \& Crochet (1997) (Third row) and simulation by Bertrand et. al. (2003) (Last row) at three different times: right column shows initial state, centre column shows the state at $120 \mathrm{~s}$ (after one cam revolution), left column shows the mixing state at $160 \mathrm{~s}$ (after $4 / 3$ cam revolutions). 
Since SPH is an explicit method, with a viscous timestep:

$$
\delta t_{\text {visc }} \approx \frac{\rho h^{2}}{\mu}=3 \times 10^{-5}
$$

the SPH simulation would need $4 \times 10^{6}$ steps to allow the cams to move through one revolution. This is impractical for what is a small-scale 2D simulation.

However, since the time-scale of the viscous forces is so small compared with the rotating cams, the viscous forces can be made significantly weaker without affecting the transport of the fluid. This will increase the time needed for the viscous forces to converge to a solution that balances the pressure and boundary forces, but this time would have to be comparable to the time-scale of the rotating cams for this to affect the transport of the fluid.

The viscosity in these simulations is set at $0.005 \mathrm{~Pa} \mathrm{~s}$, a factor of $10^{-4}$ smaller than the experiment viscosity. The simulation has been run with higher viscosities up to 0.05 $\mathrm{Pa} \mathrm{s}$, but this has no distinguishable effect on the results, indicating that the reduced viscosity has little or no impact on the solution.
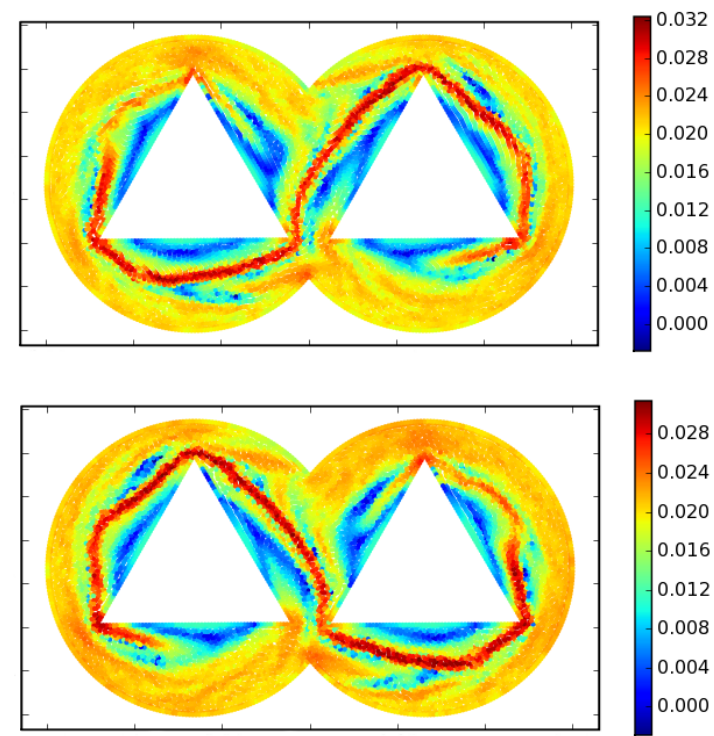

Figure 5: Top: Forward-time FTLE with $\mathrm{T}=+1$ cam revolution. Bottom: Reverse-time FTLE with $\mathrm{T}=-1$ cam revolution

\section{MIXING RESULTS}

\section{Finite-Time Lyapunov Exponents}

Figure 5 shows the results of calculating both the forward and backward time FTLE over a time interval of one cam rotation. The maxima ridges in these plots clearly show the location of the main stable and unstable manifolds and their associated fixed hyperbolic point (the intersection of the stable and unstable manifolds at the centre of the domain). The fixed point is the dominant source of mixing in the flow. Not quite as clear, but still distinguishable by comparing the two plots in Figure 5, are the many homoclinic points formed as the two manifolds criss-cross each other around the cams. A homoclinic point is the intersection of a stable and unstable manifold originating from the same hyperbolic point. As $t \rightarrow \infty$, these points will approach that hyperbolic point. The presence of transverse homoclinic points (which these points are) indicates the presence of chaotic orbits, which are essential for mixing.

Knowledge of these features is important, as they are responsible for much of the behaviour of chaotic systems and therefore the mixing. The particular geometry of these manifolds means that not all of their effects are positive. Both stable and unstable manifolds act as separatrices for the flow, i.e. there is very little flux of material across these lines. For the twin cam mixer, this means that fluid is trapped between the tangle of manifolds and the cams and does not mix with the rest of the domain. This indicates that there will be limits on the quality of the global mixing that can be produced by this specific mixer.
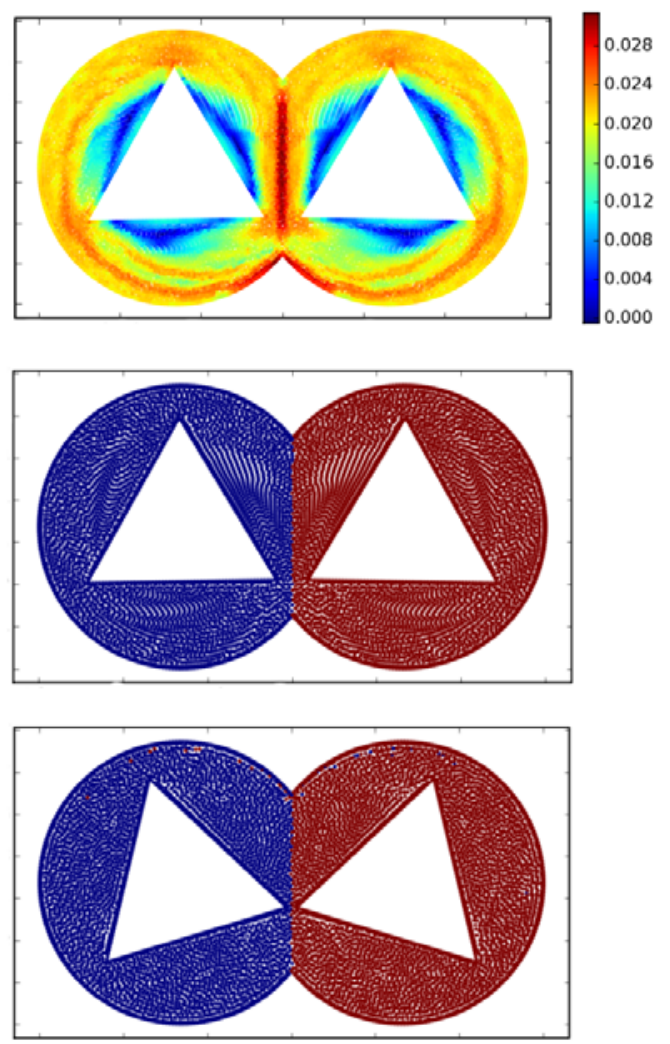

Figure 6: Counter-rotating twin cam simulation. Top: FTLE with $\mathrm{T}=+120 \mathrm{~s}$. Middle: Coloured particles at $\mathrm{t}$ $=0 \mathrm{~s}$. Bottom: Coloured particles at $\mathrm{t}=245 \mathrm{~s}$.

\section{COUNTER-ROTATING SIMULATION}

The previous simulations had the twin cams rotating in the same direction at a constant speed of $0.5 \mathrm{rpm}$. This configuration is expected to produce good mixing. For the case where the cams are rotating at the same speed but opposite directions, there should be little or no mixing. This provides an interesting case to explore what happens to the manifolds in a poor mixing case, as well as a simple test of the simulation accuracy. Figure 6 shows the initial SPH particles coloured according to their forwards and backwards-time FTLE (calculated using $T= \pm 120 \mathrm{~s}$ ). This shows a vertically orientated, strong unstable and stable manifold at $x=60 \mathrm{~mm}$. Since both manifolds coexist, every point along their length is a homoclinic point, but these are degenerate homoclinic points, which, unlike the transverse kind, do not indicate chaotic mixing. Additionally, since the manifolds are separatrices, this 
should prevent any fluid in the left chamber from entering the right and visa versa.

Figure 6 shows the particles initialised at $t=0$ so that the particles in the left chamber are a different colour to the right. It also shows the particles at $t=245 \mathrm{~s}$. The interface between the two different colours is preserved almost exactly, even though the fluid particles have been rotated though just over two periods. The small flux of particles from one chamber to the other near the top corner of the outside chamber $(x, y)=(60,55)$ (in $\mathrm{mm})$ is a physical effect. The experiment by Avalosse and Crochet (1997) showed a similar flux.

\section{CONCLUSION}

SPH is very well suited to modelling mixing processes. There is no need to numerically solve any extra equations in order to track material points or fluid concentrations, as each particle represents the same blob of fluid for the duration of the simulation. Moving objects, essential to any practical mixer, are simply modelled via moving boundary particles. Most importantly, the fluid transport is accurately modelled. The SPH simulation of a twin cam mixer compares well against experiment and two other finite-element simulations.

Traditional post-processing techniques used to analyse mixing, such as FTLE, can be used on SPH particle data. FTLE maps of the twin cam simulation have provided valuable information on the barriers to mixing in the simulation and where mixing is promoted. The Twin Cam mixer generates chaotic orbits in the outer portion of the domain, which promote mixing in this region. However, separatrices in the flow prevent fluid transport out of a region close in to the flat edges of the Cams, so this fluid area does not mix will with the rest of the domain.

\section{REFERENCES}

ARTALE, V., BOFFETTA, G., CELLANI, A., CENCINI, M. and VULPIANI, A., (1997), "Dispersion of passive tracers in closed basins: Beyond the diffusion coefficient", Physics of Fluids, 9, 3162-3171.

AVALOSSE, T. and CROCHET, M.J., (1997), "FiniteElement Simulations of Mixing: 1. Two-Dimensional Flow in Periodic Geometry", AlChE Journal, 43, No. 3, 577-587.

BERTRAND, F., THIBAULT, F., DELAMARE, L. and TANGUY, P.A., (2003), "Adaptive finite element simulation of fluid flow in twin-screw extruders", Computers and Chemical Engineering, 27, 491-500.

BERTRAND, F., TANGUY, P.A. and THIBAULT, F. (1997), "A 3D fictitious domain method for incompressible fluid flow problems", Int. J. Numerical Methods Fluids, 25, 719-736.

HALLER, G. and YUAN, G., (2000), "Lagrangian coherent structures and mixing in two-dimensional turbulence", Physica D, 147, 352-370.

HOLMES, P., (1990), "Poincare, Celestial Mechanics, Dynamical-Systems Theory and "Chaos"”, Physics Reports, 193, No. 3, 137-163.

JOSEPH, B. and LEGRAS, B., (2002), "Relation between Kinematic Boundaries, Stirring, and Barriers for the Antarctic Polar Vortex”, J. Atmos. Sci., 59, 1198-1212.

LAPEYRE, G., (2002), "Characterization of finite-time Lyapunov exponents and vectors in two-dimensional turbulence", Chaos, 12, No. 3, 688-698.
MONAGHAN, J.J., (2005), "Smoothed Particle Hydrodynamics", Rep. Prog. Phys., 68, 1703-1759.

MONAGHAN, J. J., KOS, A. and ISSA, N., (2003), "Fluid motion generated by impact", Journal of Waterway, Port, Coastal and Ocean Engineering, 129, No. 6, 250259

OTTINO, J.M., (1989), "The kinematics of mixing: stretching, chaos and transport", Cambridge University Press.

PIERREHUMBERT, R. T., and YANG, H., (1993), "Global Chaotic Mixing on Isentropic Surfaces. Journal of Atmospheric Sciences, 50, 2462-2480.

POUX, M., FAYOLLE, P., BERTRAND, J., BRIDOUX, D. and BOUSQUET, J., (1991), "Powder mixing: some practical rules applied to agitated systems", Powder Tech., 68, 213-234.

ROM-KEDAR, V., LEONARD, A. and WIGGINS, S., (1990), "An analytical study of transport, mixing and chaos in an unsteady vortical flow", J. Fluid Mech., 214, 347-394.

SHADDEN, S., (2005), "Lagrangian Coherent Structures: Analysis of time-dependent dynamical systems using finite-time Lyapunov exponents", http://www.cds.caltech.edu/ shawn/LCS-tutorial/FTLEderivation.html. 